\title{
On character amenable Banach algebras
}

\author{
by
}

\section{Z. Hu, M. Sangani Monfared and T. Traynor (Windsor, ON)}

\begin{abstract}
We obtain characterizations of left character amenable Banach algebras in terms of the existence of left $\phi$-approximate diagonals and left $\phi$-virtual diagonals. We introduce the left character amenability constant and find this constant for some Banach algebras. For all locally compact groups $G$, we show that the Fourier-Stieltjes algebra $B(G)$ is $C$-character amenable with $C<2$ if and only if $G$ is compact. We prove that if $A$ is a character amenable, reflexive, commutative Banach algebra, then $A \cong \mathbb{C}^{n}$ for some $n \in \mathbb{N}$. We show that the left character amenability of the double dual of a Banach algebra $A$ implies the left character amenability of $A$, but the converse statement is not true in general. In fact, we give characterizations of character amenability of $L^{1}(G)^{* *}$ and $A(G)^{* *}$. We show that a natural uniform algebra on a compact space $X$ is character amenable if and only if $X$ is the Choquet boundary of the algebra. We also introduce and study character contractibility of Banach algebras.
\end{abstract}

1. Introduction. Let $A$ be a Banach algebra over $\mathbb{C}$ and $\phi: A \rightarrow \mathbb{C}$ a character of $A$, that is, an algebra homomorphism from $A$ into the field of complex numbers. Let $\mathcal{M}_{\phi}^{A}$ denote the class of Banach $A$-bimodules $E$ for which the right module action of $A$ on $E$ is given by $x \cdot a=\phi(a) x$ $(a \in A, x \in E)$. Then $A$ is called left $\phi$-amenable if the first cohomology group $\mathcal{H}^{1}\left(A, E^{*}\right)$ vanishes for every $E \in \mathcal{M}_{\phi}^{A}$; furthermore, $A$ is called left character amenable if it is left $\phi$-amenable for every character $\phi$ of $A$. Similarly, one defines right $\phi$-amenable and right character amenable Banach algebras. If a Banach algebra is both left and right character amenable, it is called character amenable. The concept of (right) $\phi$-amenable Banach algebras was introduced recently by Kaniuth, Lau, and Pym in [23] (see also [24]). Character amenability was introduced independently by the second named author in [36]. Both of these concepts generalize the earlier concept of left amenability for $F$-algebras introduced by Lau in [25]. It follows from the definition that left (or right) character amenability is weaker than classical amenability introduced by Johnson in [20].

2000 Mathematics Subject Classification: 22D15, 43A20, 43A30, 43A40, 46H20, 46H25. Key words and phrases: Banach algebras, character amenability and character amenability constant, locally compact groups, uniform algebras. 
In harmonic analysis, the interest in character amenability arises from the fact that for a locally compact group $G$, the left character amenability of both the group algebra $L^{1}(G)$ and the Fourier algebra $A(G)$ is completely determined by the amenability of $G$ (see [36]).

In this paper, we continue the study of character amenable Banach algebras. The paper is organized as follows. In Section 2, we define left $\phi$ approximate and left $\phi$-virtual diagonals for a Banach algebra $A$, when $\phi$ is a nonzero character of $A$. In Theorem 2.3 , we prove that left $\phi$-amenability is equivalent to the existence of a bounded left $\phi$-approximate diagonal, which in turn is equivalent to the existence of a left $\phi$-virtual diagonal. We also define the left character amenability constant and investigate its relation with the amenability constant and the minimum bound of bounded left $\phi$-approximate diagonals.

In [13], Ghahramani, Loy, and Willis raised the question of whether amenable reflexive Banach algebras are necessarily finite-dimensional. We prove in Theorem 3.5 that if a commutative Banach algebra is reflexive, then under the weaker assumption of character amenability, the algebra must be finite-dimensional. In Section 3, we also study character amenability of the double dual of a Banach algebra. In Theorem 3.8, we show that if the double dual $A^{* *}$ of a Banach algebra $A$ (equipped with the left Arens product) is left $\phi$-amenable, then so is $A$, and in particular, the left character amenability of the double dual $A^{* *}$ implies the left character amenability of $A$. Applying this result to Lipschitz algebras $\operatorname{Lip}_{\alpha}(X)=\operatorname{lip}_{\alpha}(X)^{* *}$, we prove that when $X$ is infinite, $\operatorname{Lip}_{\alpha}(X)$ is not character amenable. The reader is referred to Kaniuth-Lau-Pym [24, Example 5.3] for the complete answer to the question for which characters $\phi$ the algebras $\operatorname{Lip}_{\alpha}(X)$ and $\operatorname{lip}_{\alpha}(X)$ are $\phi$-amenable. We show that the converse of Theorem 3.8 is not true by showing that $L^{1}(G)^{* *}$ is left character amenable if and only if the locally compact group $G$ is finite (Theorem 3.10). The dual version of Theorem 3.10 for the Fourier algebra $A(G)$ is also established (Theorem 3.12).

In Section 4, we study character amenability of the Fourier-Stieltjes algebra $B(G)$. In Theorem 4.2 , we show that $B(G)$ is $C$-character amenable with $C<2$ if and only if $G$ is compact. It is interesting to compare this result with [32, Theorem 3.2], where Runde and Spronk proved that $B(G)$ is $C$-operator amenable with $C<5$ if and only if $G$ is compact. The same example as that considered by Runde and Spronk in [33] demonstrates that there exists a noncompact locally compact group $G$ such that $B(G)$ is 2 character amenable, which shows that the dual version of [36, Corollary 2.5] proved by the second named author on the measure algebra $M(G)$ does not hold in general. 
In Section 5, we study character amenability of uniform algebras. In Theorem 5.1, we show that if a unital Banach function algebra $A$ on a compact space $X$ is character amenable, then the Choquet boundary of $A$ must coincide with $X$. As a corollary, we deduce that a natural unital uniform algebra on $X$ is character amenable if and only if its Choquet boundary coincides with $X$. It turns out that there are character amenable uniform algebras other than $C(X)$. We do not know any other type of amenability that can single out a uniform algebra other than the commutative $\mathrm{C}^{*}$-algebra $C(X)$.

In Section 6, we study the $\phi$-diagonals and their relation with character contractibility of Banach algebras.

We conclude the current section by recalling some basic terminology. For unexplained terminology and notation we refer the reader to Dales [3].

Let $A$ be a Banach algebra. We denote by $\sigma(A)$ the set of all nonzero characters of $A$. Given $\phi \in \sigma(A)$, a left $\phi$-mean is an element $\Phi$ in $A^{* *}$ such that $\Phi(\phi)=1$ and $\Phi \cdot a=\phi(a) \Phi(a \in A)$. Right $\phi$-means and (two-sided) $\phi$-means are defined similarly. The left and the right Arens products on $A^{* *}$ are denoted by $\square$ and $\diamond$, respectively. These products extend the given product on $A$ to the entire $A^{* *}$ in such a way that for every $\Phi \in A^{* *}$, the maps $\Psi \mapsto \Psi \square \Phi$ and $\Psi \mapsto \Phi \diamond \Psi$ are $w^{*}$-continuous on $A^{* *}$. Furthermore, $\Phi \in A^{* *}$ is called a mixed identity if $\Phi$ is a right identity of $\left(A^{* *}, \square\right)$ and a left identity of $\left(A^{* *}, \diamond\right)$.

Let $E$ be a Banach $A$-bimodule. A derivation $D: A \rightarrow E$ is a linear map such that $D(a b)=a \cdot D(b)+D(a) \cdot b(a, b \in A)$. Given $x \in E$, the inner derivation $\delta_{x}: A \rightarrow E$ is defined by $\delta_{x}(a)=a \cdot x-x \cdot a(a \in A)$. Let $\mathcal{Z}^{1}(A, E)$ denote the set of all continuous derivations and $\mathcal{N}^{1}(A, E)$ be the set of all inner derivations from $A$ into $E$. The first cohomology group $\mathcal{H}^{1}(A, E)$ is defined to be the quotient $\mathcal{Z}^{1}(A, E) / \mathcal{N}^{1}(A, E)$. The Banach space dual $E^{*}$ of $E$ has a natural Banach $A$-bimodule structure given by

$$
\langle a \cdot f, x\rangle=\langle f, x \cdot a\rangle, \quad\langle f \cdot a, x\rangle=\langle f, a \cdot x\rangle \quad\left(a \in A, x \in E, f \in E^{*}\right) .
$$

$A$ is called amenable if $\mathcal{H}^{1}\left(A, E^{*}\right)=\{0\}$ for all Banach $A$-bimodules $E$.

We let $\pi: A \widehat{\otimes} A \rightarrow A$ be the bounded linear map determined by $a \otimes b \mapsto a b(a, b \in A)$, where $\widehat{\otimes}$ is the projective tensor product. Note that $\|\pi\| \leq 1$.

2. Character amenability constants. We start this section with two definitions, which are variants of the concepts of approximate and virtual diagonals introduced by Johnson in [20].

Definition 2.1. Let $A$ be a Banach algebra and $\phi \in \sigma(A)$. A left [right] $\phi$-approximate diagonal for $A$ is a net $\left(m_{\alpha}\right)$ in $A \widehat{\otimes} A$ such that

(i) $\left\|m_{\alpha} \cdot a-\phi(a) m_{\alpha}\right\| \rightarrow 0\left[\left\|a \cdot m_{\alpha}-\phi(a) m_{\alpha}\right\| \rightarrow 0\right](a \in A)$;

(ii) $\left\langle\phi \otimes \phi, m_{\alpha}\right\rangle=\phi\left(\pi\left(m_{\alpha}\right)\right) \rightarrow 1$. 
Definition 2.2. Let $A$ be a Banach algebra and $\phi \in \sigma(A)$. An element $M$ of $(A \widehat{\otimes} A)^{* *}$ is a left [right] $\phi$-virtual diagonal for $A$ if

(i) $M \cdot a=\phi(a) M[a \cdot M=\phi(a) M](a \in A)$;

(ii) $\langle M, \phi \otimes \phi\rangle=\pi^{* *}(M)(\phi)=1$.

TheOREM 2.3. Let $A$ be a Banach algebra and $\phi \in \sigma(A)$. Then the following statements are equivalent:

(i) A has a left [right] $\phi$-virtual diagonal.

(ii) A has a bounded left [right] $\phi$-approximate diagonal.

(iii) $A$ is left $[$ right $] \phi$-amenable.

(iv) $A^{* *}$ has a left [right] $\phi$-mean.

Proof. The equivalence of (i) and (ii) can be shown as in the case of amenable Banach algebras. We briefly recall the arguments. (ii) implies (i), since any $w^{*}$-cluster point in $(A \widehat{\otimes} A)^{* *}$ of a bounded left [right] $\phi$-approximate diagonal for $A$ is a left [right] $\phi$-virtual diagonal for $A$. Conversely, let $M$ be a left [right] $\phi$-virtual diagonal for $A$, and let $\left(u_{\alpha}\right)$ be a net in $A \widehat{\otimes} A$ such that $M=w^{*}-\lim _{\alpha} u_{\alpha}$. Then a routine verification shows that for the net $\left(u_{\alpha}\right)$, Definition 2.1(ii) is satisfied, but the convergence in Definition 2.1(i) holds only in the weak topology on $A \widehat{\otimes} A$. Following the argument given in the proof of [3, Lemma 2.9.64], we can show that there exists a net $\left(m_{\lambda}\right)$ in $A \widehat{\otimes} A$ such that each $m_{\lambda}$ is a convex combination of elements from $\left(u_{\alpha}\right)$, and $\left(m_{\lambda}\right)$ satisfies both conditions in Definition 2.1 .

The equivalence of (iii) and (iv) is shown in [23, Theorem 1.1].

(i) implies (iv), since if $M$ is a left [right] $\phi$-virtual diagonal, then $\pi^{* *}(M)$ is a left [right] $\phi$-mean.

It remains to show that (iii) implies (i). Suppose that $A$ is left $\phi$-amenable. Consider the Banach $A$-bimodule $A \widehat{\otimes} A$ with the module actions given by

$$
a \cdot(b \otimes c)=\phi(a) b \otimes c, \quad(b \otimes c) \cdot a=b \otimes c a \quad(a, b, c \in A) .
$$

Consider the quotient Banach $A$-bimodule $E=(A \widehat{\otimes} A)^{*} / \mathbb{C} \cdot(\phi \otimes \phi)$. Let $M_{0} \in(A \widehat{\otimes} A)^{* *}$ be such that $M_{0}(\phi \otimes \phi)=1$, and let $\delta_{M_{0}}: A \rightarrow(A \widehat{\otimes} A)^{* *}$ be the inner derivation by $M_{0}$. Then the image of $\delta_{M_{0}}$ is a subset of $E^{*}$, and hence by our assumption, there exists $M_{1} \in E^{*}=\{\phi \otimes \phi\}^{\circ}$ such that $\delta_{M_{0}}=\delta_{M_{1}}$. It is easy to check that $M=M_{0}-M_{1}$ is a left $\phi$-virtual diagonal for $A$. The right side version of (iii) $\Rightarrow$ (i) can be proved similarly.

REMARK 2.4. The proof of the equivalence of (i) and (ii) in Theorem 2.3 shows that a Banach algebra has a left [right] $\phi$-virtual diagonal bounded by a constant $C$ if and only if it has a left [right] $\phi$-approximate diagonal bounded by $C$. 
Recall that a Banach algebra $A$ is left [right] 0 -amenable if and only if $A$ has a bounded left [right] approximate identity (see Johnson [20, Propositions 1.5 and 1.6]). Combining this fact with Theorem 2.3, we have

Corollary 2.5. A Banach algebra $A$ is left [right] character amenable if and only if $A$ has a bounded left [right] approximate identity and has a bounded left [right] $\phi$-approximate diagonal for every $\phi \in \sigma(A)$.

We investigate below a quantitative character amenability of Banach algebras.

Definition 2.6. Let $A$ be a Banach algebra, $C>0$ a constant, and $\phi \in \sigma(A) \cup\{0\}$. Then, $A$ is called $C$-left $\phi$-amenable if the following are satisfied:

(i) $\left(A^{* *}, \diamond\right)$ has a left identity $\Phi_{0}$ with $\left\|\Phi_{0}\right\| \leq C$ if $\phi=0$;

(ii) there exists a left $\phi$-mean $\Phi \in A^{* *}$ such that $\|\Phi\| \leq C$ if $\phi \in \sigma(A)$.

Furthermore, $A$ is called $C$-left character amenable if $A$ is $C$-left $\phi$-amenable for all $\phi \in \sigma(A) \cup\{0\}$. The least such $C$ is called the left character amenability constant of $A$.

Similarly, we can define $C$-right (resp. two-sided) $\phi$-amenability, $C$-right (resp. two-sided) character amenability, and the right (resp. two-sided) character amenability constant.

It follows from the definition that if $A$ is $C$-left or $C$-right $\phi$-amenable for some $\phi \in \sigma(A) \cup\{0\}$, then $C \geq 1$. We also note that $A$ is $C$-left 0 -amenable if and only if $A$ has a left approximate identity bounded by $C$, which is true if and only if for all $E \in \mathcal{M}_{0}^{A}$ and $D \in \mathcal{Z}^{1}\left(A, E^{*}\right), D=\delta_{g}$ for some $g \in E^{*}$ with $\|g\| \leq C\|D\|$ (see the proofs of Propositions 1.5 and 1.6 in [20]).

Theorem 2.7. Let $A$ be a Banach algebra, $C>0$ be a constant, and $\psi \in \sigma(A) \cup\{0\}$.

(i) If $A$ is $C$-left $\psi$-amenable, then, for all $E \in \mathcal{M}_{\psi}^{A}$ and $D \in \mathcal{Z}^{1}\left(A, E^{*}\right)$, there exists $g \in E^{*}$ such that $D=\delta_{g}$ and $\|g\| \leq C\|D\|$.

(ii) If for all $E \in \mathcal{M}_{\psi}^{A}$ and $D \in \mathcal{Z}^{1}\left(A, E^{*}\right)$, there exists $g \in E^{*}$ such that $D=\delta_{g}$ and $\|g\| \leq C\|D\|$, then $A$ is $C(1+2 C)$-left $\psi$-amenable.

(iii) If $\psi \neq 0$ and $A$ is $C$-left $\phi$-amenable for $\phi \in\{0, \psi\}$, then $\operatorname{ker} \psi$ has a left approximate identity of bound $2 C$.

The right side version of (i)-(iii) also holds.

Proof. (i) Let $\Psi \in A^{* *}$ with $\|\Psi\| \leq C$ be a left $\psi$-mean if $\psi \in \sigma(A)$, and a left identity of $\left(A^{* *}, \diamond\right)$ if $\psi=0$. Identifying $E$ with its natural image in $E^{* *}$, we define $g=\left.D^{* *}(\Psi)\right|_{E}$. Then a proof similar to that of [23, Theorem 1.1] shows that $D=\delta_{g}$ if $\psi \neq 0$, and $D=\delta_{-g}$ if $\psi=0$. Of course, we have $\|g\| \leq\left\|D^{* *}(\Psi)\right\| \leq C\|D\|$. 
(ii) If $\psi=0$, then, by the paragraph preceding Theorem 2.7, $A$ is $C$-left (and hence $C(1+2 C)$-left) 0 -amenable.

Let $\psi \in \sigma(A)$. Then $\|\psi\| \geq C^{-1}$. We show below that there exists a left $\psi$-mean $\Psi \in A^{* *}$ such that $\|\Psi\| \leq C(1+2 C)$. Consider $A^{*}$ being equipped with the usual left $A$-module action and with the right module action given by $f \cdot a=\psi(a) f\left(a \in A, f \in A^{*}\right)$. We define $E=A^{*} / \mathbb{C} \psi$. Then $E^{*}=$ $(\mathbb{C} \psi)^{\circ} \subseteq A^{* *}$. Using the Hahn-Banach theorem, we let $\Psi_{0} \in A^{* *}$ be such that $\Psi_{0}(\psi)=1$ and $\left\|\Psi_{0}\right\|=\|\psi\|^{-1}$. Let $\delta_{\Psi_{0}}: A \rightarrow A^{* *}, \delta_{\Psi_{0}}(a)=\psi(a) \Psi_{0}-\Psi_{0} \cdot a$, be the inner derivation by $\Psi_{0}$. It is easy to check that $\delta_{\Psi_{0}}(a) \in E^{*}$ for all $a \in A$. Thus the map $D: A \rightarrow E^{*}, D(a)=\delta_{\Psi_{0}}(a)$, is a continuous derivation (though not an inner derivation by $\Psi_{0}$, since $\Psi_{0} \notin E^{*}$ ). By our assumptions, there exists $\Psi_{1} \in E^{*}$ such that $D=\delta_{\Psi_{0}}=\delta_{\Psi_{1}}$ and $\left\|\Psi_{1}\right\| \leq C\|D\|$. We claim that $\Psi=\Psi_{0}-\Psi_{1}$ is the required left $\psi$-mean. First, $\langle\Psi, \psi\rangle=\left\langle\Psi_{0}-\Psi_{1}, \psi\right\rangle=$ $1-0=1$. Next, for all $a \in A, \delta_{\Psi_{0}}(a)=\delta_{\Psi_{1}}(a)$ implies that $\psi(a) \Psi_{0}-\Psi_{0} \cdot a=$ $\psi(a) \Psi_{1}-\Psi_{1} \cdot a$ and hence $\Psi \cdot a=\psi(a) \Psi$, proving that $\Psi$ is a left $\psi$-mean. Finally, we have

$$
\|\Psi\| \leq\left\|\Psi_{0}\right\|+C\|D\| \leq\left\|\Psi_{0}\right\|(1+2 C)=\|\psi\|^{-1}(1+2 C) \leq C(2 C+1) .
$$

(iii) Since $\left(A^{* *}, \diamond\right)$ has a left identity $\Phi_{0}$ with $\left\|\Phi_{0}\right\| \leq C, A$ has a left approximate identity of bound $C$. Let $\Psi$ be a left $\psi$-mean with $\|\Psi\| \leq C$. Then, as shown in the proof of [23, Proposition 2.2], $\Psi_{1}=\Phi_{0}-\Psi$ is a left identity of $\left((\operatorname{ker} \psi)^{* *}, \diamond\right)$ with $\left\|\Psi_{1}\right\| \leq 2 C$. Therefore, $\operatorname{ker} \psi$ has a left approximate identity of bound $2 C$.

REMARK 2.8. We note that in Theorem 2.7(iii), $\phi$ cannot be replaced by $\psi$. Also, the bound $2 C$ in Theorem 2.7(iii) is the best possible. In fact, in Example 2.10(b) below, we shall show that the Fourier algebra $A(G)$ of an amenable locally compact group $G$ is 1-character amenable. Thus if $e \in G$ is the identity element and $\tau_{e} \in \sigma(A(G))$ is the evaluation functional at $e$, then, by Theorem 2.7(iii), the closed ideal ker $\tau_{e}$ has a bounded approximate identity of bound 2. On the other hand, by Delaporte-Derighetti [5, Theorem 10], if $G$ is nondiscrete, then $\operatorname{ker} \tau_{e}$ does not have any bounded approximate identity with bound smaller than 2 (see also Kaniuth-Lau [22, Theorem 3.4], Forrest-Spronk [11, Corollary 2.3], and Derighetti [6, Theorem 12]).

Recall that a Banach algebra is $C$-amenable if it has an approximate diagonal bounded by $C$ (cf. Runde [30]).

Theorem 2.9. If $A$ is a $C$-amenable Banach algebra, then $A$ is $C$-left and $C$-right character amenable, and $C^{2}$-character amenable.

Proof. Assume that $A$ has an approximate diagonal $\left(m_{\alpha}\right)$ bounded by $C$. Let $M$ be a $w^{*}$-cluster point of $\left(m_{\alpha}\right)$ in $(A \widehat{\otimes} A)^{* *}$. Then $\pi^{* *}(M)$ is a mixed identity of $A^{* *}$ with $\left\|\pi^{* *}(M)\right\| \leq C$. 
Let $\psi \in \sigma(A)$. We define $\Psi \in A^{* *}$ by $\Psi(\lambda)=\langle M, \psi \otimes \lambda\rangle\left(\lambda \in A^{*}\right)$. Then $\|\Psi\| \leq\|M\| \leq C$. Note that $\pi^{*}(\psi)=\psi \otimes \psi$ and $\left(\pi\left(m_{\alpha}\right)\right)$ is a bounded approximate identity of $A$. We have

$$
\Psi(\psi)=\langle M, \psi \otimes \psi\rangle=\lim _{\alpha}\left\langle m_{\alpha}, \psi \otimes \psi\right\rangle=\lim _{\alpha}\left\langle\pi\left(m_{\alpha}\right), \psi\right\rangle=1 .
$$

Since $M$ is a virtual diagonal for $A$, it follows that $a \cdot M=M \cdot a$ for all $a \in A$. Therefore, for all $a \in A$ and $\lambda \in A^{*}$,

$$
\begin{aligned}
\Psi(a \cdot \lambda) & =\langle M, \psi \otimes(a \cdot \lambda)\rangle=\langle M, a \cdot(\psi \otimes \lambda)\rangle \\
& =\langle M,(\psi \otimes \lambda) \cdot a\rangle=\psi(a)\langle M, \psi \otimes \lambda\rangle=\psi(a) \Psi(\lambda) .
\end{aligned}
$$

Hence $\Psi$ is a left $\psi$-mean with norm bounded by $C$. Similarly, if we let $\Phi(\lambda)=\langle M, \lambda \otimes \psi\rangle\left(\lambda \in A^{*}\right)$, then $\Phi$ is a right $\psi$-mean with $\|\Phi\| \leq C$. Clearly, $\Phi \square \Psi$ is a two-sided $\psi$-mean satisfying $\|\Phi \square \Psi\| \leq C^{2}$.

It follows from Theorem 2.9 that for an amenable Banach algebra, the left [right] character amenability constant is less than or equal to the amenability constant. We shall see in the following examples that in general, amenability and character amenability constants are not equal.

ExAmples 2.10. (a) By Theorem 2.9, if $A$ is an amenable Banach algebra with amenability constant 1 , then $A$ has character amenability constant 1 as well. Therefore, all commutative $\mathrm{C}^{*}$-algebras and group algebras $L^{1}(G)$ with $G$ amenable have character amenability constant 1.

(b) Let $G$ be a locally compact group, $1<p<\infty$, and $A_{p}(G)$ be the generalized Fourier algebra of $G$, as introduced by Figà-Talamanca and by Herz. By Herz [18, Theorem 6], if $G$ is amenable, then $A_{p}(G)$ has a bounded approximate identity of bound 1 , and hence $A_{p}(G)^{* *}$ has a mixed identity of norm 1. In [34, Lemma 3.1], it is proved that for each $\phi \in \sigma\left(A_{p}(G)\right)$, $A_{p}(G)^{* *}$ has a $\phi$-mean of norm 1 . Thus, if $G$ is amenable, then $A_{p}(G)$ has character amenability constant 1 . In particular, taking $p=2$, we deduce that the Fourier algebra $A(G)$ has character amenability constant 1 if $G$ is amenable.

In [27, Theorem 4.9], Lau, Loy, and Willis showed that for certain finite groups $G$ of order $2^{2 n+1}$, the amenability constant of $A(G)$ is greater than $2^{[n / 2]}$. Thus, the difference between the amenability constant and the character amenability constant can be as large as we like. Furthermore, it is known that there are compact groups $G$ such that $A(G)$ is not amenable (see Johnson [21]). Therefore, there are Banach algebras $A$ such that $A$ is not amenable while the character amenability constant of $A$ remains minimal $(=1)$.

(c) The Banach algebra $\mathcal{B}(\mathcal{H})$ of all continuous linear operators on a separable Hilbert space $\mathcal{H}$ with $\operatorname{dim} \mathcal{H} \geq 2$ is unital such that $\sigma(\mathcal{B}(\mathcal{H}))=\emptyset$. Hence $\mathcal{B}(\mathcal{H})$ is 1 -character amenable. 
(d) It is easy to find Banach algebras with large character amenability constants. In fact, $l_{n}^{1}$ with pointwise multiplication has character amenability constant $n$.

Theorem 2.11. Let $A$ be a Banach algebra. Let $C \geq 1$ and let $\left(A_{\alpha}\right)$ be a net of closed, $C$-left [right] character amenable subalgebras of $A$ such that

(i) $A_{\alpha} \subseteq A_{\beta}$ whenever $\alpha \leq \beta$;

(ii) $\bigcup_{\alpha} A_{\alpha}$ is dense in $A$.

Then $A$ is $C$-left [right] character amenable.

Proof. Note that for each $\alpha, A_{\alpha}^{* *}=\bar{A}_{\alpha}^{w^{*}} \subseteq A^{* *}$. If $\psi \in \sigma(A)$, then $\psi_{\alpha}=\left.\psi\right|_{A_{\alpha}} \in \sigma\left(A_{\alpha}\right) \cup\{0\}$, and we must have $\psi_{\alpha} \neq 0$ for all $\alpha$ large enough. For each $\alpha$ with $\psi_{\alpha} \neq 0$, let $\Psi_{\alpha} \in A_{\alpha}^{* *}$ be a left $\psi_{\alpha}$-mean with $\left\|\Psi_{\alpha}\right\| \leq C$. Let $\Psi \in A^{* *}$ be a $w^{*}$-cluster point of $\left(\Psi_{\alpha}\right)$. Without loss of generality, we may assume that $w^{*}-\lim _{\alpha} \Psi_{\alpha}=\Psi$. Then it is easy to verify that $\Psi$ is a left $\psi$-mean with $\|\Psi\| \leq C$. The verification that $\left(A^{* *}, \diamond\right)$ has a left identity with norm bounded by $C$ is routine.

The Banach algebra $l^{2}$ (with pointwise product) does not have a bounded (left) approximate identity and hence is not (left) character amenable. However, for each $n \geq 1, l_{n}^{2}$ is a $\sqrt{n}$-character amenable subalgebra of $l^{2}$, and the family $\left(l_{n}^{2}\right)$ satisfies conditions (i) and (ii) in Theorem 2.11. It follows that in this theorem, one cannot relax the condition of $C$-left character amenability (for a fixed $C$ ) on the family $\left(A_{\alpha}\right)$, even if one only wants to have the character amenability of $A$.

The proof of the following lemma is routine and is omitted.

Lemma 2.12. Let $A$ and $B$ be Banach algebras.

(i) If $\mu: A \rightarrow B$ is a continuous homomorphism with a dense range in $B$, then the $C$-left [right] character amenability of $A$ implies that $B$ is $\|\mu\| C$-left [right] character amenable.

(ii) $A \oplus_{\infty} B$ is $C$-left [right] character amenable if and only if both $A$ and $B$ are $C$-left [right] character amenable.

(iii) If $A$ is $C_{1}$-left [right] character amenable and $B$ is $C_{2}$-left [right $]$ character amenable, then for $1 \leq p<\infty, A \oplus_{p} B$ is $\left(C_{1}^{p}+C_{2}^{p}\right)^{1 / p}$-left [right] character amenable.

The following corollary is a consequence of Theorem 2.11 and Lemma 2.12 .

Corollary 2.13. Let $\left(A_{\alpha}\right)$ be a family of Banach algebras. Then $c_{0}-\bigoplus_{\alpha} A_{\alpha}$ is C-left [right] character amenable if and only if each $A_{\alpha}$ is $C$-left [right] character amenable. 
To close this section, we consider the relation between the left character amenability constant and the minimum bound for left approximate (or virtual) diagonals. For a given Banach algebra $A$ with $\sigma(A) \neq \emptyset$, we define $C_{v}=\inf \{C: \forall \phi \in \sigma(A), \exists$ a left $\phi$-virtual diagonal with norm $\leq C\}$; $C_{a}=\inf \{C: \forall \phi \in \sigma(A), \exists$ a left $\phi$-approximate diagonal with norm $\leq C\}$; $C_{m}=\inf \{C: \forall \phi \in \sigma(A), \exists$ a left $\phi$-mean with norm $\leq C\}$.

It follows from Remark 2.4 that $C_{v}=C_{a}$. If $M$ is a left $\phi$-virtual diagonal with $\|M\| \leq C$, then $\pi^{* *}(M)$ is a left $\phi$-mean and $\left\|\pi^{* *}(M)\right\| \leq C$. Therefore, we have $C_{m} \leq C_{v}=C_{a}$.

TheOREM 2.14. If $A$ is a $C$-left character amenable Banach algebra and $\sigma(A) \neq \emptyset$, then $C_{v} \leq C^{2}(1+2 C)$.

Proof. Let $\left(e_{\alpha}\right)_{\alpha \in I}$ be a bounded left approximate identity of $A$ with $\left\|e_{\alpha}\right\| \leq C$. Then $\left(e_{\alpha} \otimes e_{\beta}\right)_{(\alpha, \beta) \in I \times I}$ is a bounded left approximate identity of $A \widehat{\otimes} A$ bounded by $C^{2}$. Given $\phi \in \sigma(A)$, we have $\|\phi \otimes \phi\| \geq 1 / C^{2}$. To obtain a left $\phi$-virtual diagonal for $A$ with norm bounded by $C^{2}(2 C+1)$, we argue as in the proof of (iii) $\Rightarrow$ (i) in Theorem 2.3. Keeping the same notation and using the Hahn-Banach theorem, we choose $M_{0} \in(A \widehat{\otimes} A)^{* *}$ such that $M_{0}(\phi \otimes \phi)=1$ and $\left\|M_{0}\right\|=\|\phi \otimes \phi\|^{-1} \leq C^{2}$. Our assumption together with Theorem 2.7(i) then implies that there exists $M_{1} \in E^{*}$ with $\delta_{M_{0}}=\delta_{M_{1}}$ and $\left\|M_{1}\right\| \leq C\left\|\delta_{M_{0}}\right\| \leq 2 C^{3}$, where $E=(A \widehat{\otimes} A)^{*} / \mathbb{C} \cdot(\phi \otimes \phi)$. It follows that $M=M_{0}-M_{1}$ is a left $\phi$-virtual diagonal for $A$ and

$$
\|M\| \leq\left\|M_{0}\right\|+\left\|M_{1}\right\| \leq C^{2}(1+2 C) .
$$

3. Character amenability of double duals. Let $A$ be a Banach algebra. In this section, we study the relation between character amenability of $A$ and that of its double dual $A^{* *}$. We start with a few preliminary results regarding ideals in $A$.

Recall that a subspace $E$ of $A$ is called weakly left [right] invariantly complemented in $A$ if $E^{\circ}=\left\{\lambda \in A^{*}:\left.\lambda\right|_{E}=0\right\}$ is left [right] invariantly complemented in $A^{*}$, that is, if there exists a left [right] $A$-module homomorphism $P: A^{*} \rightarrow E^{\circ}$ such that $P^{2}=P$.

For convenience, we collect two known lemmas below. The first lemma is due to Forrest (see [9, Proposition 6.4]).

Lemma 3.1. Let $A$ be a Banach algebra and let I be a closed right ideal in $A$.

(i) If I has a bounded left approximate identity, then I is weakly left invariantly complemented in A. 
(ii) If A has a bounded left approximate identity and I is weakly left invariantly complemented in $A$, then I has a bounded left approximate identity.

The corresponding assertions hold if the words "left" and "right" are interchanged in the above statements.

Lemma 3.2. Let $A$ be a left character amenable Banach algebra and let $I$ be a closed ideal in $A$. Then the following statements are equivalent:

(i) I has a bounded left approximate identity.

(ii) I is weakly left invariantly complemented in A.

(iii) I is left character amenable.

The right side version of the lemma is also true.

Proof. The equivalence of (i) and (ii) follows from Lemma 3.1. And the equivalence of (i) and (iii) is proved in [36, Theorem 2.6(ii)].

LEMma 3.3. If $A$ is a character amenable commutative Banach algebra, then every closed ideal of finite codimension in $A$ is character amenable.

Proof. Let $I$ be an ideal in $A$ with $\operatorname{dim}(A / I)=n$. There exists a chain of ideals

$$
I=I_{0} \triangleleft I_{1} \triangleleft \cdots \triangleleft I_{n}=A
$$

such that each $I_{i-1}$ is a closed ideal in $I_{i}$ and $\operatorname{dim}\left(I_{i} / I_{i-1}\right)=1$ for $i=$ $1, \ldots, n$. Since $I_{n-1}$ is an ideal in $A$ of codimension 1 , it can be easily verified that either $I_{n-1}$ is a modular ideal and hence $I_{n-1}=\operatorname{ker} \phi$ for some $\phi \in$ $\sigma(A)$, or $A^{2} \subseteq I_{n-1}$ (cf. [3, Proposition 1.3.37]). The latter case is impossible, since $A$ has a bounded approximate identity and hence $A^{2}=A$ by Cohen's factorization theorem. Thus $I_{n-1}$ is a maximal modular ideal and has a bounded approximate identity by [36, Corollary 2.7]. By Lemma 3.2, $I_{n-1}$ is character amenable. The proof can be completed by an induction.

REMARK 3.4. It follows from Lemma 3.3 and Dales [3, Corollary 2.9.36] that every finite-dimensional Banach algebra extension of a character amenable commutative Banach algebra $A$ splits strongly. This result was obtained by the second named author in [36, Corollary 3.2] using a different method.

In [13], Ghahramani, Loy, and Willis showed that an amenable Banach algebra which is a Hilbert space is finite-dimensional. They asked whether this result holds for reflexive Banach algebras. In our next result, we consider a related question in the commutative case, under the weaker assumption of character amenability.

We recall that a modular annihilator algebra is a semiprime algebra in which every maximal modular left ideal has a nonzero right annihilator (cf. Palmer [28, Definition 8.4.6]). 
TheOREM 3.5. Let $A$ be a character amenable, reflexive, commutative Banach algebra. Then $A \cong \mathbb{C}^{n}$ for some $n \in \mathbb{N}$.

Proof. Since $A$ has a bounded approximate identity and is reflexive, it has an identity $1_{A}$. We shall show that $A$ is a semisimple modular annihilator algebra, and hence is finite-dimensional by [28, Theorem 8.4.14]. Then $A$ is isomorphic to $\mathbb{C}^{n}$ by Wedderburn's structure theorem.

Let $M$ be a maximal ideal in $A$. Then $M=\operatorname{ker} \phi$ for some $\phi \in \sigma(A)$, and therefore $M$ has a bounded approximate identity since $A$ is character amenable (see [36, Corollary 2.7]). Since $M$ is reflexive, $M$ has an identity $1_{M}$. It follows that $1_{A}-1_{M}$ is a nonzero annihilator of $M$.

Note that $A / \operatorname{rad} A$ is a commutative, unital, semisimple, reflexive Banach algebra which is character amenable. Our argument in the previous paragraph shows that $A / \operatorname{rad} A$ must be a semisimple modular annihilator algebra and hence must be finite-dimensional. Thus $\operatorname{rad} A$ is of finite codimension in $A$. By Lemma 3.3, $\operatorname{rad} A$ is character amenable and hence must be unital, since it is reflexive. This implies that $\operatorname{rad} A=\{0\}$. We have shown that $A$ is a unital, semisimple modular annihilator algebra, completing the proof of the theorem.

Let ${ }_{\phi} \mathcal{M}^{A}$ denote the class of Banach $A$-bimodules $E$ for which the left module action of $A$ on $E$ is given by $a \cdot x=\phi(a) x(a \in A, x \in E)$. Replacing the class of Banach $A$-bimodules by ${ }_{\phi} \mathcal{M}^{A}$, we have the following analogue of a result of Gourdeau [14] on amenable Banach algebras (cf. Dales [3, Proposition 2.8.59(i)]).

Lemma 3.6. Let $A$ be a Banach algebra and $\phi \in \sigma(A) \cup\{0\}$. Then the following statements are equivalent:

(i) $A$ is left $[$ right $] \phi$-amenable.

(ii) $\mathcal{H}^{1}\left(A, E^{* *}\right)=\{0\}$ for every $E \in{ }_{\phi} \mathcal{M}^{A}\left[E \in \mathcal{M}_{\phi}^{A}\right]$.

(iii) Given $E \in{ }_{\phi} \mathcal{M}^{A}\left[E \in \mathcal{M}_{\phi}^{A}\right]$, every continuous derivation $D: A \rightarrow E$ is approximately inner; that is, there exists a bounded net $\left(x_{\alpha}\right)$ in E such that

$$
D(a)=\|\cdot\|-\lim _{\alpha}\left(a \cdot x_{\alpha}-x_{\alpha} \cdot a\right) \quad(a \in A) .
$$

Note that $E \in{ }_{\phi} \mathcal{M}^{A}$ (resp. $E \in \mathcal{M}_{\phi}^{A}$ ) if and only if $E^{*} \in \mathcal{M}_{\phi}^{A}$ (resp. $\left.E^{*} \in{ }_{\phi} \mathcal{M}^{A}\right)$. The arguments used in the proof for the classical case can be applied here so that we omit the details (cf. [3, Proposition 2.8.59]).

REMARK 3.7. We would like to point out the following fact related to Lemma 3.6(iii). If $A$ is $C$-left character amenable, then it can be seen that the net $\left(x_{\alpha}\right)$ determining a derivation $D: A \rightarrow E$ as in Lemma 3.6(iii) satisfies $\left\|x_{\alpha}\right\| \leq C\|D\|$. On the other hand, if $A$ is a $C$-amenable unital Banach algebra with an approximate diagonal $\left(t_{i}\right)$ bounded by $C$, then, as 
noted by Johnson $[21$, p. 361], there exists a constant $N$ such that for any $A$-bimodule $E$ and any continuous derivation $D: A \rightarrow E$, the net $\left(x_{\alpha}\right)$ in $E$ determining $D$ satisfies $\left\|x_{\alpha}\right\| \leq N\|D\|$; however, $N$ is related to the bound of the net $\left(1 \otimes 1-t_{i}\right)$.

The amenable version of the result below on Banach algebras has been proved by Gourdeau [15], and by Ghahramani, Loy, and Willis [13]. In view of Lemma 3.6, the proof for the amenable case carries over without difficulty.

Theorem 3.8. Let $A$ be a Banach algebra and $\phi \in \sigma(A) \cup\{0\}$. If $\left(A^{* *}, \square\right)$ is left $[$ right $] \phi$-amenable, then $A$ is left $[$ right $] \phi$-amenable. In particular, if $\left(A^{* *}, \square\right)$ is left $[$ right $]$ character amenable, then so is $A$.

Let $0<\alpha<1$ and $X$ be a compact metric space. Consider the Lipschitz algebra $\operatorname{Lip}_{\alpha}(X)$ and its closed subalgebra $\operatorname{lip}_{\alpha}(X)$ (cf. Dales [3]). If $X$ is a finite space, then $\operatorname{lip}_{\alpha}(X)=\operatorname{Lip}_{\alpha}(X)$ is semisimple and finite-dimensional, and hence is amenable. However, if $X$ is infinite and $x_{0} \in X$ is not an isolated point of $X$, then by [3, Theorem 4.4.30(iv)], $M_{x_{0}}=\left\{f \in \operatorname{lip}_{\alpha}(X)\right.$ : $\left.f\left(x_{0}\right)=0\right\}$ does not have any bounded approximate identity, and hence by Lemma 3.3, $\operatorname{lip}_{\alpha}(X)$ is not character amenable. Since $\operatorname{lip}_{\alpha}(X)^{* *}=\operatorname{Lip}_{\alpha}(X)$, as an application of Theorem 3.8, we see that $\operatorname{Lip}_{\alpha}(X)$ is not character amenable when $X$ is infinite.

We point out that the question for which characters $\phi$ the algebras $\operatorname{Lip}_{\alpha}(X)$ and $\operatorname{lip}_{\alpha}(X)$ are $\phi$-amenable has been clarified in [24]. Kaniuth, Lau, and Pym [24, Example 5.3] showed that for $0<\alpha \leq 1, \operatorname{Lip}_{\alpha}(X)$ is $\phi_{x}$-amenable if and only if $x$ is an isolated point of $X$, and this equivalence holds for $\operatorname{lip}_{\alpha}(X)$ with $0<\alpha<1$, where $x \mapsto \phi_{x}$ is the canonical homeomorphism between $X$ and $\sigma\left(\operatorname{Lip}_{\alpha}(X)\right)\left(\operatorname{resp} . \sigma\left(\operatorname{lip}_{\alpha}(X)\right)\right)$. See [24, Example 5.3] for details on these facts.

We recall that if $A$ is a Banach algebra, then a closed $A$-submodule $X$ of $A^{*}$ is called left introverted if $\Phi \square \lambda \in X$ for all $\Phi \in A^{* *}$ and $\lambda \in X$. In this case, $X^{*}$ is a Banach algebra with the multiplication induced by the left Arens product $\square$ on $A^{* *}$.

The following theorem is analogous to Lau-Loy [26, Corollary 4.4] on weak amenability of $X^{*}$, where $X$ is a left introverted subspace of $L^{\infty}(G)$ of a locally compact abelian group $G$. Inspired by the proof given by Lau and Loy, we show the character amenability version for the class of maximally almost periodic groups (MAP-groups). It is known that the class of MAPgroups contains compact groups, finite extensions of abelian groups, free groups, and the discrete Heisenberg group. It is also known that a direct product of two MAP-groups is MAP, and for almost connected groups, MAP $=$ SIN (groups with small invariant neighborhoods). See Palmer [29] for more information on MAP-groups. 
THEOREM 3.9. Let $G$ be a maximally almost periodic locally compact group. Let $X$ be a left introverted subspace of $L^{\infty}(G)$ containing $\operatorname{AP}(G)$ (the space of almost periodic functions on $G$ ). Then $X^{*}$ is left character amenable if and only if $G$ is finite.

Proof. If $G$ is finite, then $G$ is amenable, $X=L^{\infty}(G)$, and hence $X^{*}=$ $L^{\infty}(G)^{*}=L^{1}(G)$ is left character amenable (see [36, Corollary 2.4]).

Conversely, suppose that $X^{*}$ is left character amenable. Then $\operatorname{AP}(G)^{*}$ is also left character amenable, since the restriction map $X^{*} \rightarrow \operatorname{AP}(G)^{*}, \Phi \mapsto$ $\left.\Phi\right|_{\mathrm{AP}(G)}$, is a continuous surjective homomorphism. Since $\mathrm{AP}(G) \cong C(b G)$, where $b G$ is the Bohr compactification of $G$, it follows that $M(b G) \cong \operatorname{AP}(G)^{*}$ is left character amenable. By [36, Corollary 2.5], $b G$ must be discrete, and hence $b G$ is finite. Since for a maximally almost periodic group $G$, the canonical homomorphism from $G$ into $b G$ is injective, it follows that $G$ is finite.

Examples of left introverted subspaces of $L^{\infty}(G)$ containing $\operatorname{AP}(G)$ include $\operatorname{AP}(G), \operatorname{WAP}(G)$ (weakly almost periodic functions on $G$ ), $L U C(G)$ (bounded left uniformly continuous functions on $G$ ), and of course $L^{\infty}(G)$. The condition $\operatorname{AP}(G) \subseteq X$ is essential in order to conclude that $G$ is finite. For example, if $G$ is noncompact and $X=C_{0}(G)$ (in which case $X \cap \operatorname{AP}(G)=\{0\})$, then $X^{*}=M(G)$ is character amenable if and only if $G$ is discrete and amenable.

As proved in [36, Corollary 2.4], the character amenability of $L^{1}(G)$ and the character amenability of $A(G)$ are each equivalent to the amenability of $G$. We show below that for all locally compact groups $G$, the character amenability of $L^{1}(G)^{* *}$ and the character amenability of $A(G)^{* *}$ are each equivalent to the finiteness of $G$. As a consequence, we see that in general the converse of Theorem 3.8 is not true.

The following result is the character amenable version of GhahramaniLoy-Willis [13, Theorem 1.3] for the locally compact group case, which shows that $\left(L^{1}(G)^{* *}, \square\right)$ is amenable if and only if $G$ is finite.

TheOREM 3.10. Let $G$ be a locally compact group. Then $\left(L^{1}(G)^{* *}, \square\right)$ is character amenable if and only if $G$ is finite.

Proof. We need to prove the necessity part of the theorem. Suppose that $L^{1}(G)^{* *}$ is character amenable. Then $L^{1}(G)^{* *}$ does not have any nonzero continuous point derivation corresponding to any character $\phi \in \sigma\left(L^{1}(G)^{* *}\right)$. It follows from [4, Theorem 11.17] that $G$ is finite.

Lemma 3.11. Let $A$ be a Banach algebra such that $\left(A^{* *}, \square\right)$ has a bounded left approximate identity. Then $A^{*}=\left\langle A^{*} \cdot A\right\rangle$ (the closed linear span of $\left.A^{*} \cdot A\right)$. 
Proof. Suppose that $\left(A^{* *}, \square\right)$ has a bounded left approximate identity. Then $\left(A^{* *}, \square\right)$ has a left identity $E$. Let $\left(a_{\alpha}\right)$ be a net in $A$ such that $a_{\alpha} \rightarrow E$ in the $w^{*}$-topology on $A^{* *}$. Let $f \in A^{*}$. Then, $f \cdot a_{\alpha} \rightarrow f$ weakly in $A^{*}$. Thus, $f \in\left\langle A^{*} \cdot A\right\rangle$. Therefore, $A^{*}=\left\langle A^{*} \cdot A\right\rangle$.

In [17], Granirer proved that for all locally compact groups $G,\left(A(G)^{* *}, \square\right)$ is amenable if and only if $G$ is finite. We now show the character amenable version of this result by Granirer.

TheOREM 3.12. Let $G$ be a locally compact group. Then $\left(A(G)^{* *}, \square\right)$ is character amenable if and only if $G$ is finite.

Proof. We only have to prove the necessity part. Suppose that $A(G)^{* *}$ is character amenable. By Lemma 3.11, $\operatorname{VN}(G)=\langle\mathrm{VN}(G) \cdot A(G)\rangle=\mathrm{UC}(\widehat{G})$, where $\operatorname{UC}(\widehat{G})$ is the space of all uniformly continuous functionals on $A(G)$. By [16, Theorem 3], $G$ must be compact. We show below that $G$ is discrete and hence $G$ is finite.

Let $H$ be a closed abelian subgroup of $G$. Then the restriction map $r: A(G) \rightarrow A(H)$ is a surjective norm decreasing homomorphism (see [18]) and so is its second adjoint $r^{* *}: A(G)^{* *} \rightarrow A(H)^{* *}$. By [36, Theorem 2.6(i)], $A(H)^{* *}$ is character amenable. However, $A(H)^{* *} \cong L^{1}(\widehat{H})^{* *}$, where $\widehat{H}$ is the dual group of $H$. By Theorem 3.10, $\widehat{H}$ is finite, so that $H$ is also finite.

From the proof of [10, Theorem 3.2], one can see that $G$ is totally disconnected. The argument given in [10] is as follows. Let $G_{0}$ be the connected component of $e$. Since $G$ is periodic, $G_{0}$ and any homomorphic image of $G_{0}$ are also periodic. For any neighborhood $U$ of $e$ in $G_{0}$, there exists a compact normal subgroup $N$ of $G_{0}$ such that $N \subseteq U$ and $G_{0} / N$ is a periodic connected Lie group. Thus, $G_{0} / N$ is trivial. This is true for all neighborhoods $U$ of $e$ in $G_{0}$. Therefore, $G_{0}$ is trivial.

Finally, we follow the proof of [26, Lemma 6.5] as given below to conclude that $G$ is discrete. Let $H$ be a compact open subgroup of $G$; by [19, Theorem 7.7], such $H$ exist in any open neighborhood of $e$ in $G$. If $H$ is infinite, then $H$ would contain an infinite abelian subgroup by [39, Theorem 2], which is impossible. Therefore, $H$ is a finite open subgroup of $G$ and hence $G$ is discrete.

4. Character amenability of Fourier-Stieltjes algebras. Let $G$ be a locally compact group. The Fourier-Stieltjes algebra $B(G)$ of $G$ introduced by Eymard [8] is the collection of all coefficient functions of continuous unitary representations of $G$. The set $B(G)$ can be identified with the dual space of the group $\mathrm{C}^{*}$-algebra $C^{*}(G)$. With the norm defined by this duality 
and the pointwise multiplication, $B(G)$ is a Banach algebra containing $A(G)$ as a closed ideal. We have $B(G)=A(G)$ if and only if $G$ is compact. The dual of $B(G)$ is the enveloping von Neumann algebra of $C^{*}(G)$, and is denoted by $W^{*}(G)$. For more information on $B(G)$ and $W^{*}(G)$, the reader is referred to $[7,8,38]$.

If $G$ is an abelian group and $\widehat{G}$ is its dual group, it follows from Bochner's theorem that $B(G)$ is isometrically algebra isomorphic to the measure algebra $M(\widehat{G})$ of $\widehat{G}$ via the Fourier-Stieltjes transform. The second-named author proved in [36, Corollary 2.5] that for all locally compact groups $G$, $M(G)$ is (1-)left character amenable if and only if $G$ is discrete and amenable. A natural question is whether the dual version of this equivalence holds, that is, whether the compactness of $G$ is necessary to ensure the character amenability of $B(G)$.

In $[32,33]$, Runde and Spronk studied the operator amenability of $B(G)$. They proved that $B(G)$ is $C$-operator amenable with $C<5$ if and only if $G$ is compact (see [32, Theorem 3.2]). In [33], they further showed that the upper bound 5 for the constant $C$ cannot be improved. More precisely, they considered the following Fourier-Stieltjes algebra. Let $n \geq 1$ be an integer, $p$ a prime number, $\mathbb{Q}_{p}$ the field of $p$-adic numbers, and $\mathbb{Z}_{p}$ the ring of $p$-adic integers. Then the semidirect product $G_{p, n}=\mathbb{Q}_{p}^{n} \ltimes \mathrm{GL}\left(n, \mathbb{Z}_{p}\right)$ (with the group action given by $\left.(v, T)\left(v^{\prime}, T^{\prime}\right)=\left(v+T v^{\prime}, T T^{\prime}\right)\right)$ is a noncompact amenable locally compact group. Let $q: G_{p, n} \rightarrow G_{n, p} / \mathbb{Q}_{p}^{n} \cong \mathrm{GL}\left(n, \mathbb{Z}_{p}\right)$ be the quotient map, and let

$$
A\left(\operatorname{GL}\left(n, \mathbb{Z}_{p}\right)\right) \circ q=\left\{u \circ q: u \in A\left(\operatorname{GL}\left(n, \mathbb{Z}_{p}\right)\right)\right\} .
$$

Runde and Spronk showed that

$$
B\left(G_{p, n}\right)=\left[A\left(\mathrm{GL}\left(n, \mathbb{Z}_{p}\right)\right) \circ q\right] \oplus_{1} A\left(G_{p, n}\right),
$$

and $B\left(G_{p, n}\right)$ is operator amenable with operator amenability constant 5 (see [33, Proposition 2.1 and Theorem 2.3]).

By Eymard [8, Corollaire 2.26(3)], the map $u \mapsto u \circ q$ is an isometric algebra isomorphism between $B\left(\mathrm{GL}\left(n, \mathbb{Z}_{p}\right)\right)$ and its image $B\left(\mathrm{GL}\left(n, \mathbb{Z}_{p}\right)\right) \circ q$. Thus, $A\left(\mathrm{GL}\left(n, \mathbb{Z}_{p}\right)\right)$ is isomorphic to $A\left(\mathrm{GL}\left(n, \mathbb{Z}_{p}\right)\right) \circ q$. Since both $\operatorname{GL}\left(n, \mathbb{Z}_{p}\right)$ and $G_{p, n}$ are amenable groups, it follows from Example 2.10(b) and Lemma 2.12(iii) that $B\left(G_{p, n}\right)$ is 2-character amenable. This example shows that $B(G)$ can be character amenable when $G$ is noncompact. Therefore, we do not have the dual version of the result on the character amenability of $M(G)$.

Our objective in this section is to show that $B(G)$ is $C$-character amenable with $C<2$ if and only if $G$ is compact. We note that the proof of Theorem 3.2 in Runde-Spronk [32] does not carry over to our case, since 
among other reasons, $C$-character amenability is defined in terms of bounded approximate identity and $\phi$-means rather than approximate diagonals.

In our proof, we use the following decomposition of $B(G)$ proved by Runde and Spronk in [32, Section 2], which can be considered as a dual version to the decomposition $M(G)=l^{1}(G) \oplus_{1} M_{c}(G)$.

Lemma 4.1 ([32]). If $G$ is a noncompact locally compact group, then there are central projections $P_{\mathcal{F}}, P_{\mathcal{P} \mathcal{I F}}$ in $W^{*}(G)$ such that

(i) $P_{\mathcal{F}} \in \sigma(B(G))$;

(ii) $A_{\mathcal{F}}(G)=P_{\mathcal{F}} \cdot B(G)$ is a closed subalgebra of $B(G)$ containing the constant function $1_{G}$;

(iii) $A_{\mathcal{P I F}}(G)=P_{\mathcal{P I F}} \cdot B(G)$ is a closed ideal of $B(G)$ containing $A(G)$;

(iv) $B(G)=A_{\mathcal{F}}(G) \oplus_{1} A_{\mathcal{P I F}}(G)$.

TheOREM 4.2. Let $G$ be a locally compact group. Then the following statements are equivalent:

(i) $G$ is compact;

(ii) $B(G)$ is 1-character amenable;

(iii) $B(G)$ is $C$-character amenable for some constant $C$ with $C<2$.

Proof. If $G$ is compact, then $B(G)=A(G)$ is 1-character amenable (see Example 2.10(b)). Therefore, (i) implies (ii). Obviously, (ii) implies (iii).

It remains to show that (iii) implies (i). Suppose that $G$ is not compact but $B(G)$ is $C$-character amenable. Note that $P_{\mathcal{F}} \in \sigma(B(G))$. Let $\Phi_{\mathcal{F}} \in$ $B(G)^{* *}$ be any $P_{\mathcal{F}}$-mean. We show that $\left\|\Phi_{\mathcal{F}}\right\| \geq 2$, which implies that $C \geq 2$, completing the proof of the theorem.

Consider the Banach space direct sums

$$
W^{*}(G)=A_{\mathcal{F}}(G)^{*} \oplus_{\infty} A_{\mathcal{P} \mathcal{I} \mathcal{F}}(G)^{*}, \quad W^{*}(G)^{*}=A_{\mathcal{F}}(G)^{* *} \oplus_{1} A_{\mathcal{P I} \mathcal{F}}(G)^{* *},
$$

which follow directly from Lemma 4.1(iv). Clearly, $P_{\mathcal{F}} \in A_{\mathcal{F}}(G)^{*}$ and $P_{\mathcal{P} \mathcal{I F}}$ $\in A_{\mathcal{P I F}}(G)^{*}$. If we write $\Phi_{\mathcal{F}}=\Phi_{1}+\Phi_{2}$ with $\Phi_{1} \in A_{\mathcal{F}}(G)^{* *}$ and $\Phi_{2} \in$ $A_{\mathcal{P} \mathcal{I} \mathcal{F}}(G)^{* *}$, then

$$
1=\left\langle\Phi_{\mathcal{F}}, P_{\mathcal{F}}\right\rangle=\left\langle\Phi_{1}+\Phi_{2}, P_{\mathcal{F}}\right\rangle=\left\langle\Phi_{1}, P_{\mathcal{F}}\right\rangle .
$$

This implies that $\left\|\Phi_{1}\right\| \geq 1$.

Let $\omega: G \rightarrow W^{*}(G)$ be the universal representation of $G$. Then $\omega(e)=$ $P_{\mathcal{F}}+P_{\mathcal{P} \mathcal{I} \mathcal{F}}$ is the identity of $W^{*}(G)$ and $\langle\omega(e), u\rangle=u(e)(u \in B(G))$. Choose $u \in A(G)$ such that $u(e)=1$. Then $u \cdot \omega(e)=\omega(e)$. Since $A(G) \subseteq A_{\mathcal{P I F}}(G)$ and $P_{\mathcal{F}} \in A_{\mathcal{F}}(G)^{*}$, we have $P_{\mathcal{F}}(u)=0$. It follows that $\left\langle\Phi_{1}, P_{\mathcal{F}}\right\rangle+\left\langle\Phi_{2}, P_{\mathcal{P} \mathcal{F} \mathcal{F}}\right\rangle=\left\langle\Phi_{\mathcal{F}}, \omega(e)\right\rangle=\left\langle\Phi_{\mathcal{F}}, u \cdot \omega(e)\right\rangle=P_{\mathcal{F}}(u)\left\langle\Phi_{\mathcal{F}}, \omega(e)\right\rangle=0$. Thus, $\left\langle\Phi_{2}, P_{\mathcal{P} \mathcal{I} \mathcal{F}}\right\rangle=-\left\langle\Phi_{1}, P_{\mathcal{F}}\right\rangle=-1$ and hence $\left\|\Phi_{2}\right\| \geq 1$. Therefore, $\left\|\Phi_{\mathcal{F}}\right\|=\left\|\Phi_{1}\right\|+\left\|\Phi_{2}\right\| \geq 2$. 
Remarks 4.3. (a) Note that $A_{\mathcal{F}}(G) \cong B(b G)=A(b G)$ (see [32, Proposition 2.1]). Therefore, for all locally compact groups $G, A_{\mathcal{F}}(G)$ is always character amenable. It follows from [36, Theorem 2.6(v)] that $B(G)$ is character amenable if $A_{\mathcal{P I F}}(G)$ is character amenable.

(b) The group $G_{p, n}$ shows that there exists a noncompact amenable locally compact group $G$ such that $B(G)$ is 2-character amenable. On the other hand, for all noncompact locally compact abelian groups $G, B(G) \cong$ $M(\widehat{G})$ is not character amenable.

5. Character amenability of uniform algebras. We briefly recall the terminology needed in this section. For more details, the reader is referred to Dales [3, Chapter 4].

Let $X$ be a topological space. A function algebra $A$ on $X$ is a subalgebra of $\mathbb{C}^{X}$ that strongly separates points of $X$ for which the $A$-topology on $X$ coincides with the given topology. A Banach function algebra on $X$ is a function algebra on $X$ which is a Banach algebra with respect to some norm; a uniform algebra on $X$ is a function algebra on $X$ which is a closed subalgebra of $\left(C^{b}(X),|\cdot|_{X}\right)$, where $|\cdot|_{X}$ is the supremum norm on $C^{b}(X)$. A Banach function algebra is called natural if $\sigma(A)=\left\{\tau_{x}: x \in X\right\}$, where $\tau_{x}$ is the evaluation functional at $x$.

Suppose that $X$ is a compact space and $A$ is a unital Banach function algebra on $X$. The Choquet boundary of $A$, denoted by $\Gamma_{0}(A)$, consists of all those $x \in X$ for which $\delta_{x}$ is the unique probability measure $\mu$ on $X$ such that $f(x)=\int_{X} f d \mu$ for every $f \in A$. The following theorem relates the character amenability to the Choquet boundary.

THeOREM 5.1. If $A$ is a character amenable unital Banach function algebra on a compact space $X$, then $\Gamma_{0}(A)=X$.

Proof. Given $x \in X, \tau_{x}$-amenability implies that $\operatorname{ker} \tau_{x}$ has a bounded approximate identity (see [23, Proposition 2.2]). A slight modification of the proof of [2, Lemma 1.6.3] shows that there exist $0<\alpha<\beta<1$ such that for each neighborhood $U$ of $x$, there exists $f \in A$ satisfying

$$
|f|_{X} \leq 1, \quad f(x)>\beta, \quad|f|_{X \backslash U}<\alpha .
$$

It is known that under these conditions, we have $x \in \Gamma_{0}(A)$ (cf. [2, Theorem 2.2.1]).

For uniform algebras $A$, the condition that $x \in \Gamma_{0}(A)$ is equivalent to the existence of a bounded approximate identity of $\operatorname{ker} \tau_{x}$ (cf. Dales [3, Theorem 4.3.5]), which implies that $A$ is $\tau_{x}$-amenable (see Kaniuth-Lau-Pym [23, Proposition 2.1]). Thus, the Choquet boundary completely characterizes the character amenability of a natural unital uniform algebra. 
COROLlary 5.2. A natural unital uniform algebra $A$ on a compact space $X$ is character amenable if and only if $\Gamma_{0}(A)=X$.

It is perhaps not surprising that the Choquet boundary alone cannot characterize the character amenability of a general Banach function algebra, since the definition of Choquet boundary does not involve the norm of the algebra. For a specific example, let $G=\mathrm{SL}(2, \mathbb{R})$ (or any nonamenable locally compact group). Let $A(G)^{\#}$ be the unitization of $A(G)$, and $G_{\infty}$ the onepoint compactification of $G$. Then $A(G)^{\#}$ can be identified with a unital Banach function algebra on $G_{\infty}$ with $\sigma\left(A^{\#}(G)\right)=\left\{\tau_{x}: x \in G_{\infty}\right\} \cong G_{\infty}$. Since $G$ is not amenable, $A(G)$ and hence $A(G)^{\#}$ are not character amenable (see Sangani-Monfared [36, Corollary 2.4]). However, one can get $\Gamma_{0}\left(A^{\#}(G)\right)=$ $G_{\infty}$ by showing that for each $x \in G_{\infty}$ and each neighborhood $U$ of $x$ in $G_{\infty}$, there exists $f \in A^{\#}(G)$ such that

$$
|f|_{G_{\infty}} \leq 1, \quad f(x)=1, \quad|f|_{G_{\infty} \backslash U}=0 .
$$

If $x \in G$, the construction of such a function $f$ is well-known (see, for example, Kaniuth-Lau-Pym [23, Example 2.6]). If $x=\infty, K \subseteq G$ is compact, and $U=G_{\infty} \backslash K$ is an open neighborhood of $\infty$, then we can choose an open neighborhood $V$ of $K$ in $G$, and find a function $f \in A(G)$ such that $0 \leq f \leq 1,\left.f\right|_{K}=1$, and $\left.f\right|_{G \backslash V}=0$. Then the function $g=1-f \in A(G)^{\#}$ satisfies $|g|_{G_{\infty}} \leq 1, g(\infty)=1$, and $|g|_{G_{\infty} \backslash U}=0$. In fact, since $G=\operatorname{SL}(2, \mathbb{R})$ is metrizable, each $\{x\}$ is a $G_{\delta}$-set in $G_{\infty}$ and therefore each $x \in G_{\infty}$ is a peak point for $A^{\#}\left(G_{\infty}\right)$ (cf. Dales [3, p. 447]).

By Sheinberg's theorem, it is known that a unital uniform algebra $A$ on a compact space $X$ is amenable if and only if $A=C(X)$ (cf. [3, Theorem 5.6.2]). Recently, Runde [31] showed an operator space version of Sheinberg's theorem when a uniform algebra is equipped with its minimal operator space structure and amenability is replaced by operator amenability. It is natural to ask whether amenability in Sheinberg's theorem can be replaced by character amenability. The following example due to Basener $[1$, Theorem 3] shows that this is not the case.

For a compact set $K \subseteq \mathbb{C}$, let $R(K)$ be the uniform algebra of all functions on $K$ which are uniform limits of rational functions with poles outside $K$. Let $K$ be a compact subset of the unit disk $D$ such that the only Jensen measures for $R(K)$ are trivial, and $R(K) \neq C(K)$ (cf., for example, Stout [37, Section 24]). Now let

$$
\Omega_{K}=\left\{(z, w) \in \mathbb{C}^{2}: z \in K,|z|^{2}+|w|^{2}=1\right\} .
$$

For this $\Omega_{K}$, Basener [1] showed that $R\left(\Omega_{K}\right) \neq C\left(\Omega_{K}\right)$. However, given $\left(z_{0}, w_{0}\right) \in \Omega_{K}$, the polynomial $P(z, w)=\left(\overline{z_{0}} z+\overline{w_{0}} w+1\right) / 2$ peaks at $\left(z_{0}, w_{0}\right)$ and hence $\Gamma_{0}\left(R\left(\Omega_{K}\right)\right)=\Omega_{K}$. Therefore, by Corollary $5.2, R\left(\Omega_{K}\right)$ is a char- 
acter amenable Banach algebra. We remark that since $\sigma\left(R\left(\Omega_{K}\right)\right)=\Omega_{K}$ (cf. Dales [3, Proposition 4.3.12(iii)]), $R\left(\Omega_{K}\right) \neq C(X)$ for any compact space $X$.

Let $K$ be a compact subset of $\mathbb{C}$ and let $P(K)$ be the uniform algebra on $K$ consisting of uniform limits of polynomials. It is interesting that for such uniform algebras, the character amenable version of Sheinberg's result holds. We start with a general result that was kindly pointed out to us by the referee.

Lemma 5.3. Let $K$ be a compact subset of $\mathbb{C}^{n}$ for some $n \geq 1$, and let $P(K)$ be character amenable. Then $K$ has empty interior and is polynomially convex.

Proof. Since $P(K)$ is character amenable, it does not have any nonzero point derivations. Thus $K$ must have an empty interior, since for any $w$ in the interior of $K$ and for any $1 \leq k \leq n, f \mapsto \frac{d f}{d z_{k}}(w)$ is a nonzero point derivation on $P(K)$.

Let $\widehat{K}$ be the polynomially convex hull of $K$. Then $P(K)$ is isometrically isomorphic to $P(\widehat{K})$ (cf. for example [12, p. 27]; the argument for $n>1$ is the same). Therefore, by Theorem 5.1, $\widehat{K}=\Gamma_{0}(P(\widehat{K})) \subseteq \Gamma_{0}(P(K))=K$. That is, $K$ is polynomially convex.

TheOREM 5.4. For a compact subset $K$ of $\mathbb{C}$, the uniform algebra $P(K)$ is character amenable if and only if $P(K)=C(K)$.

Proof. The "only if" part of the theorem needs a proof. By Lemma 5.3, $K$ must be polynomially convex and must have empty interior. Hence by Lavrentiev's theorem (cf. [12, Theorem II.8.7]), we must have $P(K)=C(K)$.

6. Character contractibility. In the final section of this paper, we consider character contractibility of Banach algebras. We start with the following definition.

Definition 6.1. Let $A$ be a Banach algebra and $\phi \in \sigma(A)$. A left [right] $\phi$-diagonal for $A$ is an element $m$ of $A \widehat{\otimes} A$ such that

(i) $m \cdot a=\phi(a) m[a \cdot m=\phi(a) m] \quad(a \in A)$;

(ii) $\langle\phi \otimes \phi, m\rangle=\phi(\pi(m))=1$.

If $m$ is both left and right $\phi$-diagonal, it is called a $\phi$-diagonal.

It is easy to see that if $m$ is a left $\phi$-diagonal and $n$ is a right $\phi$-diagonal, then $n m$ is a (two-sided) $\phi$-diagonal. No direct relation seems to exist between a projective diagonal and a $\phi$-diagonal. However, we will see that the existence of a projective diagonal implies the existence of $\phi$-diagonals for every $\phi \in \sigma(A)$. 
Definition 6.2. Let $A$ be a Banach algebra and $\phi \in \sigma(A) \cup\{0\}$. Then $A$ is called left $\phi$-contractible if $\mathcal{H}^{1}(A, E)=\{0\}$ for all $E \in{ }_{\phi} \mathcal{M}^{A}$. Furthermore, $A$ is called left character contractible if it is left $\phi$-contractible for all $\phi \in \sigma(A) \cup\{0\}$.

Similar definitions hold for right $\phi$-contractible and right character contractible Banach algebras.

$A$ is called character contractible if it is both left and right character contractible.

Theorem 6.3. Let $A$ be a Banach algebra and $\phi \in \sigma(A)$.

(i) A is left [right] 0-contractible if and only if $A$ has a left [right] identity.

(ii) $A$ is left [right] $\phi$-contractible if and only if $A$ has a left [right] $\phi$-diagonal.

(iii) $A$ is left [right] character contractible if and only if $A$ has a left $[$ right $]$ identity and has a left [right $] \phi$-diagonal for every $\phi \in \sigma(A)$.

Proof. We only prove the left version of the theorem. Similar arguments will establish the right side version.

(i) Suppose that $A$ is left 0 -contractible. Let $E=A \oplus_{1} A$ be the Banach $A$-bimodule with the module actions given by $a \cdot(b, c)=(0,0)$ and $(b, c)$. $a=(b a, c a)(a, b, c \in A)$. By our assumption, the continuous derivation $D: A \rightarrow E, D(a)=(a, a)$, must be inner and hence $D=\delta_{\left(a_{0}, b_{0}\right)}$ for some $\left(a_{0}, b_{0}\right) \in E$. It follows that both $-a_{0}$ and $-b_{0}$ are left identities for $A$. The converse is obvious and its proof is omitted.

(ii) Let $A$ be left $\phi$-contractible. Consider the Banach $A$-bimodule $A \widehat{\otimes} A$ with the module actions given by $a \cdot(b \otimes c)=\phi(a) b \otimes c$ and $(b \otimes c) \cdot a=$ $b \otimes c a(a, b, c \in A)$. Let $m_{0} \in A \widehat{\otimes} A$ be such that $\left\langle\phi \otimes \phi, m_{0}\right\rangle=1$, and consider the inner derivation

$$
\delta_{m_{0}}: A \rightarrow A \widehat{\otimes} A, \quad a \mapsto a \cdot m_{0}-m_{0} \cdot a=\phi(a) m_{0}-m_{0} \cdot a .
$$

Note that the image of $\delta_{m_{0}}$ is a subset of $\operatorname{ker}(\phi \otimes \phi)$. Hence, by the assumption, there exists $m_{1} \in \operatorname{ker}(\phi \otimes \phi)$ such that $\delta_{m_{0}}=\delta_{m_{1}}$. Then $m_{0}-m_{1}$ is a left $\phi$-diagonal for $A$.

Conversely, let $m$ be a left $\phi$-diagonal for $A$, let $E \in{ }_{\phi} \mathcal{M}^{A}$, and let $D: A \rightarrow E$ be a continuous derivation. Let $x_{0}=D(\pi(m))$. It suffices to show that $D a=\delta_{x_{0}} a$ for all $a \in A$ satisfying $\phi(a)=1$ (since these elements $\operatorname{span} A)$. Let $a \in A$ with $\phi(a)=1$. Then

$$
\delta_{x_{0}} a=x_{0}-x_{0} \cdot a=D(\pi(m))-[D(\pi(m) a)-\pi(m) \cdot D a]=D a,
$$

where the last equality holds since $\pi(m) a=\pi(m \cdot a)=\phi(a) \pi(m)=\pi(m)$.

(iii) This is immediate by (i) and (ii). 
Our next result is analogous to Corollary 2.3 in Kaniuth-Lau-Pym [23], where they proved that a Banach algebra $A$ is left [right] $\phi$-amenable and has a bounded left [right] approximate identity if and only if ker $\phi$ has a bounded left [right] approximate identity.

Theorem 6.4. Let $A$ be a Banach algebra and $\phi \in \sigma(A)$. Then $A$ is left [right $] \phi$-contractible and has a left [right] identity if and only if $\operatorname{ker} \phi$ has a left [right] identity.

Proof. To prove the necessity of the condition, we note that by Theorem 6.3(ii), $A$ has a left $\phi$-diagonal $m \in A \widehat{\otimes} A$. If $e$ is a left identity of $A$, then $e-\pi(m)$ is a left identity of $\operatorname{ker} \phi$.

It remains to prove the sufficiency of the condition. Let $e^{\prime}$ be a left identity of ker $\phi$. Take $a_{0} \in A$ such that $\phi\left(a_{0}\right)=1$. Then $e=a_{0}+e^{\prime}-e^{\prime} a_{0}$ is a left identity of $A$ and $m=e \otimes\left(e-e^{\prime} e\right)$ is a left $\phi$-diagonal for $A$. To verify the last assertion, we note that

$$
\phi(\pi(m))=\phi(e)-\phi\left(e^{\prime}\right) \phi(e)=\phi(e)=1 \quad \text { and } \quad m \cdot e=m .
$$

Furthermore, if $a \in A$, then $a=(a-\phi(a) e)+\phi(a) e$ with $a-\phi(a) e \in \operatorname{ker} \phi$. Hence we have

$$
m \cdot(a-\phi(a) e)=e \otimes\left((a-\phi(a) e)-e^{\prime}(a-\phi(a) e)\right)=e \otimes 0=0 .
$$

Therefore,

$$
m \cdot a=m \cdot(a-\phi(a) e)+\phi(a) m \cdot e=\phi(a) m .
$$

The right side version of the theorem can be proved similarly.

Clearly, every left [right] character contractible Banach algebra is left [right] character amenable. The converse, however, is not true. For example, if $G$ is an amenable, noncompact locally compact group, then the Fourier algebra $A(G)$ is character amenable (see [36, Corollary 2.4]) but not character contractible since it is not unital. It is seen from the definitions that every contractible Banach algebra is character contractible. The converse is not true. For instance, if $\mathcal{H}$ is a separable infinite-dimensional Hilbert space, then $\sigma(\mathcal{B}(\mathcal{H}))=\emptyset$, and hence $\mathcal{B}(\mathcal{H})$ is character contractible, but $\mathcal{B}(\mathcal{H})$ is not contractible (cf. Runde [30, Corollary 6.1.8]).

EXAMPLE 6.5. To give an example of $\phi$-diagonals, we consider the Banach algebra $\mathcal{A}$ of all upper-triangular $3 \times 3$ matrices over $\mathbb{C}$. We have $\sigma(\mathcal{A})=\left\{\phi_{1}, \phi_{2}, \phi_{3}\right\}$, where

$$
\phi_{k}\left(\left[a_{i j}\right]\right)=a_{k k}, \quad k=1,2,3 .
$$

The verification of the following assertions is routine. 
(i) $\mathcal{A}$ is right $\phi_{1}$-contractible and

$$
M_{1}=\left[\begin{array}{lll}
1 & 0 & 0 \\
0 & 0 & 0 \\
0 & 0 & 0
\end{array}\right] \otimes\left[\begin{array}{lll}
1 & 0 & 0 \\
0 & 1 & 0 \\
0 & 0 & 1
\end{array}\right]
$$

is a right $\phi_{1}$-diagonal for $\mathcal{A}$.

(ii) $\mathcal{A}$ is not left $\phi_{1}$-amenable, since ker $\phi_{1}$ has no left identity.

(iii) $\mathcal{A}$ is neither left nor right $\phi_{2}$-amenable, since ker $\phi_{2}$ has neither a left nor a right identity.

(iv) $\mathcal{A}$ is left $\phi_{3}$-contractible, and

$$
M_{3}=\left[\begin{array}{lll}
1 & 0 & 0 \\
0 & 1 & 0 \\
0 & 0 & 1
\end{array}\right] \otimes\left[\begin{array}{lll}
0 & 0 & 0 \\
0 & 0 & 0 \\
0 & 0 & 1
\end{array}\right]
$$

is a left $\phi_{3}$-diagonal for $\mathcal{A}$.

(v) $\mathcal{A}$ is not right $\phi_{3}$-amenable, since ker $\phi_{3}$ has no right identity.

Let $A$ be a Banach algebra and $A^{\text {op }}$ the opposite algebra of $A$. Every Banach $A$-bimodule $E$ has a canonical $A^{\text {op }}$-bimodule structure given by $a \circ x=x \cdot a$ and $x \circ a=a \cdot x\left(a \in A^{\mathrm{op}}, x \in E\right)$. For the definition of higher order cohomology groups of Banach algebras, the reader is referred to Dales [3, p. 274]. The following lemma is well known.

Lemma 6.6. For every Banach A-bimodule E and every positive integer $n$,

$$
\mathcal{H}^{n}(A, E) \cong \mathcal{H}^{n}\left(A^{\mathrm{op}}, E\right)
$$

via the isomorphism $T \mapsto T^{\mathrm{o}}$, where $T^{\mathrm{o}}\left(a_{1}, \ldots, a_{n}\right)=T\left(a_{n}, \ldots, a_{1}\right)$.

Corollary 6.7. Let $A$ be a Banach algebra and $\phi \in \sigma(A) \cup\{0\}$.

(i) $A$ is left $[$ right $] \phi$-contractible if and only if $A^{\mathrm{op}}$ is right $[$ left $] \phi$-contractible.

(ii) $A$ is left [right] $\phi$-amenable if and only if $A^{\text {op }}$ is right [left $] \phi$ character contractible.

(iii) If $A$ is left [right $] \phi$-contractible, then $\mathcal{H}^{n}(A, E)=\{0\}$ for all $E \in$ ${ }_{\phi} \mathcal{M}^{A}\left[E \in \mathcal{M}_{\phi}^{A}\right]$ and all positive integers $n$.

(iv) If $A$ is left $[$ right $] \phi$-amenable, then $\mathcal{H}^{n}\left(A, E^{*}\right)=\{0\}$ for all $E \in$ $\mathcal{M}_{\phi}^{A}\left[E \in{ }_{\phi} \mathcal{M}^{A}\right]$ and all positive integers $n$.

Parts (i) and (ii) are immediate consequences of Lemma 6.6. In (iii) and (iv), the statements for the "left" case follow from the standard reduction of dimension formula $\mathcal{H}^{n+1}(A, E)=\mathcal{H}^{1}\left(A,\left(\mathcal{B}^{n}(A, E), \star\right)\right)$ as proved 
for example in Dales [3, Proposition 2.8.22]. Then the "right" case in (iii) and (iv) follows by applying Lemma 6.6.

Our objective in the remainder of this section is to show that a character contractible commutative Banach algebra is isomorphic to $\mathbb{C}^{n}$ for some $n \in \mathbb{N}$. We start with a few preliminary results.

We recall that if $A$ and $B$ are Banach algebras and $\theta \in \sigma(B)$, then the $\theta$-Lau product $A \times{ }_{\theta} B$ is the space $A \times B$ equipped with the multiplication

$$
(a, b) \cdot\left(a^{\prime}, b^{\prime}\right)=\left(a a^{\prime}+\theta(b) a^{\prime}+\theta\left(b^{\prime}\right) a, b b^{\prime}\right),
$$

and the norm $\|(a, b)\|=\|a\|+\|b\|$. Then $A \times_{\theta} B$ is a Banach algebra, $A$ is a closed two-sided ideal in $A \times_{\theta} B$, and $\left(A \times_{\theta} B\right) / A \cong B$. We note that if $B=\mathbb{C}$ and $\theta$ is the identity map on $\mathbb{C}$, then $A \times{ }_{\theta} \mathbb{C}=A^{\#}$ is the unitization of $A$. In [35, Proposition 2.4] it is shown that

$$
\sigma\left(A \times{ }_{\theta} B\right)=\sigma(A) \times\{\theta\} \cup\{0\} \times \sigma(B) .
$$

For more information on these algebras, the reader is referred to $[25,35]$.

The following result can be obtained by modifying the proofs of [36, Theorem 2.6 and Proposition 2.8] given for the character amenable case.

Lemma 6.8. Let $A$ and $B$ be Banach algebras and $I$ a closed two-sided ideal of $A$.

(i) If $A$ is left [right] character contractible and $\mu: A \rightarrow B$ is a continuous algebra homomorphism such that $\overline{\mu(A)}=B$, then $B$ is left [right] character contractible.

(ii) If $A$ is left [right] character contractible, then I is left [right] character contractible if and only if I has a left [right] identity.

(iii) Given $\theta \in \sigma(B), A \times_{\theta} B$ is left [right] character contractible if and only if both $A$ and $B$ are left [right] character contractible.

(iv) $A \times B$ is left [right] character contractible if and only if both $A$ and $B$ are left $[$ right $]$ character contractible.

In view of Lemma 6.8, the following two results can be proved by simple modifications of the arguments used in the proofs of Lemma 3.3 and Theorem 3.5.

Lemma 6.9. If $A$ is a character amenable commutative Banach algebra, then every ideal of finite codimension in $A$ is character contractible.

THEOREM 6.10. If $A$ is a character contractible commutative Banach algebra, then $A \cong \mathbb{C}^{n}$ for some $n \in \mathbb{N}$. 
REMARK 6.11. Theorem 6.10 is not true for noncommutative character contractible Banach algebras, as the example $\mathcal{B}(\mathcal{H})$ shows, where $\mathcal{H}$ is an infinite-dimensional separable Hilbert space. Another example can be given by using Lemma $6.8(\mathrm{iii})$. If $\theta \in \sigma\left(\mathbb{C}^{n}\right)$, then $\mathcal{B}(\mathcal{H}) \times{ }_{\theta} \mathbb{C}^{n}$ is an infinitedimensional character contractible Banach algebra which has nonempty spectrum. Note that $\mathcal{B}(\mathcal{H}) \times_{\theta} \mathbb{C}^{n}$ is an infinite-dimensional character contractible Banach algebra whose maximal ideals are of finite codimension; such examples do not exist among contractible Banach algebras (cf. Runde [30, Proposition 4.1.2]).

Acknowledgements. We would like to thank Brian Forrest, Volker Runde, and Nico Spronk for their helpful suggestions regarding the material in Section 4. We are grateful to the referee for various valuable comments and suggestions to improve the quality of the paper, in particular, the suggestions on the present enhanced formulation of Theorem 2.7 and the result stated in Lemma 5.3.

The first and the second authors were partially supported by NSERC.

\section{References}

[1] R. F. Basener, On rationally convex hulls, Trans. Amer. Math. Soc. 182 (1973), 353-381.

[2] A. Browder, Introduction to Function Algebras, W. A. Benjamin, New York, 1969.

[3] H. G. Dales, Banach Algebras and Automatic Continuity, Oxford Univ. Press, Oxford, 2000.

[4] H. G. Dales, A. T.-M. Lau, and D. Strauss, Banach algebras on semigroups and their compactifications, Mem. Amer. Math. Soc., to appear.

[5] J. Delaporte and A. Derighetti, Best bounds for the approximate units for certain ideals of $L^{1}(G)$ and of $A_{p}(G)$, Proc. Amer. Math. Soc. 124 (1996), 1159-1169.

[6] A. Derighetti, Conditional expectations on $C V_{p}(G)$. Applications, J. Funct. Anal. 247 (2007), 231-251.

[7] J. Ernest, A new group algebra for locally compact groups, Amer. J. Math. 86 (1964), 467-492.

[8] P. Eymard, L'algèbre de Fourier d'un groupe localement compact, Bull. Soc. Math. France 92 (1964), 181-236.

[9] B. Forrest, Amenability and bounded approximate identities in ideals of $A(G)$, Illinois J. Math. 34 (1990), 1-25.

[10] - Weak amenability and the second dual of the Fourier algebra, Proc. Amer. Math. Soc. 125 (1997), 2373-2378.

[11] B. Forrest and N. Spronk, Best bounds for approximate identities in ideals of the Fourier algebra vanishing on subgroups, ibid. 134 (2006), 111-116.

[12] T. W. Gamelin, Uniform Algebras, Chelsea, New York, 1984.

[13] F. Ghahramani, R. J. Loy, and G. A. Willis, Amenability and weak amenability of second conjugate Banach algebras, Proc. Amer. Math. Soc. 124 (1996), 1489-1497. 
[14] F. Gourdeau, Amenability of Lipschitz algebras, Math. Proc. Cambridge Philos. Soc. 112 (1992), 581-588.

[15] -, Amenability and the second dual of Banach algebras, Studia Math. 125 (1997), $75-81$.

[16] E. E. Granirer, On group representations whose $C^{*}$-algebra is an ideal in its von Neumann algebra, Ann. Inst. Fourier (Grenoble) 29 (1979), no. 4, 37-52.

[17] - A survey on some functional analytic properties of the Fourier algebra $A(G)$ of a locally compact group, Southeast Asian Bull. Math. 20 (1996), no. 2, 1-12.

[18] C. Herz, Harmonic synthesis for subgroups, Ann. Inst. Fourier (Grenoble) 23 (1973), no. $3,91-123$.

[19] E. Hewitt and K. A. Ross, Abstract Harmonic Analysis I, Springer, New York, 1979.

[20] B. E. Johnson, Cohomology in Banach Algebras, Mem. Amer. Math. Soc. 127 (1972).

[21] - Non-amenability of the Fourier algebra of a compact group, J. London Math. Soc. 50 (1994), 361-374.

[22] E. Kaniuth and A. T.-M. Lau, A separation property of positive definite functions on locally compact groups and applications to Fourier algebras, J. Funct. Anal. 175 (2000), 89-110.

[23] E. Kaniuth, A. Lau, and J. Pym, On $\phi$-amenability of Banach algebras, Math. Proc. Cambridge Philos. Soc. 144 (2008), 85-96.

[24] - - - - - On character amenability of Banach algebras, J. Math. Anal. Appl. 344 (2008), 942-955.

[25] A. T.-M. Lau, Analysis on a class of Banach algebras with applications to harmonic analysis on locally compact groups and semigroups, Fund. Math. 118 (1983), $161-175$.

[26] A. T.-M. Lau and R. J. Loy, Weak amenability of Banach algebras on locally compact groups, J. Funct. Anal. 145 (1997), 175-204.

[27] A. T.-M. Lau, R. J. Loy, and G. A. Willis, Amenability of Banach and $C^{*}$-algebras on locally compact groups, Studia Math. 119 (1996), 161-178.

[28] T. W. Palmer, Banach Algebras and the General Theory of *-Algebras, Vol. I, Cambridge Univ. Press, Cambridge, 1994.

[29] —, Banach Algebras and the General Theory of *-Algebras, Vol. II, Cambridge Univ. Press, Cambridge, 2001.

[30] V. Runde, Lectures on Amenability, Springer, New York, 2002.

[31] —, The operator amenability of uniform algebras, Canad. Math. Bull. 46 (2003), 632-634.

[32] V. Runde and N. Spronk, Operator amenability of Fourier-Stieltjes algebras, Math. Proc. Cambridge Philos. Soc. 136 (2004), 675-686.

[33] - , -, Operator amenability of Fourier-Stieltjes algebras II, Bull. London Math. Soc. 39 (2007), 194-202.

[34] M. Sangani Monfared, Extensions and isomorphims for the generalized Fourier algebras of a locally compact group, J. Funct. Anal. 198 (2003), 413-444.

[35] - On certain products of Banach algebras with applications to harmonic analysis, Studia Math. 178 (2007), 277-294.

[36] —, Character amenability of Banach algebras, Math. Proc. Cambridge Philos. Soc. 144 (2008), 697-706.

[37] E. L. Stout, The Theory of Uniform Algebras, Bogden and Quigley, New York, 1971.

[38] M. E. Walter, $W^{*}$-algebras and nonabelian harmonic analysis, J. Funct. Anal. 11 (1972), 17-38. 
[39] E. I. Zelmanov, On periodic compact groups, Israel J. Math. 77 (1992), 83-95.

Department of Mathematics and Statistics

University of Windsor

Windsor, ON, Canada N9B 3P4

E-mail: zhiguohu@uwindsor.ca

monfared@uwindsor.ca

tt@uwindsor.ca

Received July 17, 2008

Revised version January 15, 2009

$(6386)$ 